\title{
Altered homotopic connectivity in postherpetic neuralgia: a resting state $\mathrm{fMRI}$ study
}

This article was published in the following Dove Press journal:

Journal of Pain Research

31 October 2016

Number of times this article has been viewed

Jian Jiangl,*
Lili Gu',*
Dan Bao
Shunda Hong'
Wei He'
Yonming Tan'
Xianjun Zeng'
Honghan Gong'
Daying Zhang'
Fuqing Zhou'
'Department of Radiology,
'Department of Pain, The First
Affiliated Hospital, Nanchang
University, Nanchang, Jiangxi,
People's Republic of China
*These authors contributed equally to
this work

this work
Correspondence: Fuqing Zhou Department of Radiology, The First Affiliated Hospital, Nanchang University, 17 Yongwaizhen Street, Nanchang 330006, Jiangxi, People's Republic of China

Tel +86 79l 88695132

Emailfq.chou@yahoo.com
Objective: The aim of this study was to explore interhemispheric intrinsic connectivity in patients with postherpetic neuralgia (PHN).

Methods: We obtained resting-state functional magnetic resonance imaging data from 18 righthanded PHN patients (11 males, 7 females; mean age, 59.67 \pm 8.41 years) and 18 well-matched healthy controls (11 males, 7 females; mean age, $38.50 \pm 7.51$ years). Interhemispheric connectivity was examined using voxel-mirrored homotopic connectivity (VMHC), and seed-based functional connectivity analysis was performed.

Results: Compared with the healthy controls, the patients with PHN showed abnormally decreased homotopic connectivity in the dorsolateral prefrontal cortex and the precuneus and posterior cingulate cortex (PCUN/PCC). The decreased VMHC in the PCUN/PCC was positively correlated with the visual analog scale of $\mathrm{PHN}$ in the PHN patient group $(\rho=0.651 ; P=0.006)$. Receiver operating characteristic (ROC) analysis revealed that the areas under the curves for the two brain regions were 0.898 for the prefrontal cortex and 0.923 for the PCUN/PCC, which indicated that the VMHC could be used to discriminate PHN patients from healthy controls. A subsequent seed-based functional connectivity analysis revealed widely disrupted intrinsic connectivity between the regions that showed local homotopic connectivity deficits and the areas subserving the default-mode network.

Conclusion: Our results indicated reduced interhemispheric functional connectivity in patients with PHN, which seems to be an important new avenue to investigate to better understand the nature of disconnection of the functional architecture in patients with PHN.

Keywords: voxel-mirrored homotopic connectivity, postherpetic neuralgia, functional magnetic resonance imaging, resting state, pain

\section{Introduction}

Postherpetic neuralgia (PHN), which is defined as typical chronic neuropathic pain that develops after healed herpes zoster infection and persists for $>3$ months, significantly impacts patient quality of life. ${ }^{1,2}$ PHN can exist with various neuropathic signs that are associated with irreparable damage to the peripheral nervous system. ${ }^{2-4}$ Recently, several neuroimaging studies ${ }^{5-9}$ have explored possible alterations of structure and functions in patients with PHN pain, including anatomical damage in the bilateral insula and superior temporal gyrus $;{ }^{5}$ functional aberrant activation involved in affective responses, sensory discrimination (the thalamus, primary and secondary somatosensory, insula, and anterior cingulated cortices), and emotion; ${ }^{6,7}$ increased functional connectivity of the bilateral putamen ${ }^{7}$ and decreased local efficiency of the putamen. ${ }^{8}$ Assessment of cerebral blood flow (CBF) in these regions has provided an 
understanding of the underlying physiological basis, ${ }^{9}$ with results showing increased $\mathrm{CBF}$ in the striatum, thalamus, primary somatosensory cortex (S1), insula, amygdala, and inferior parietal lobule, and decreased $\mathrm{CBF}$ in the frontal cortex. ${ }^{9}$ However, anatomical and functional neuroimaging studies on the central nervous systems of PHN patients remain limited.

The changes in the functional activity of the brain during PHN are not fully understood at present. Magnetic resonance imaging (MRI) technologies developed for noninvasive human brain mapping have been used to expose alterations of functional patterns and have provided new insights into the potential neural mechanisms of neuropathic pain in patients with PHN. Interhemispheric homotopic connectivity has been suggested to be a basic principle of the brain's functional architecture in integrated brain functions that underlie coherent cognition, behavior, and consciousness. ${ }^{10,11}$ Voxel-mirrored homotopic connectivity (VMHC) is a datadriven voxel-based intrinsic functional connectivity (iFC) approach used to characterize the high degree of synchrony in spontaneous activity between geometrically corresponding interhemispheric regions. ${ }^{10}$ This approach differs from analyses of the regional activity intensity (amplitude of low-frequency fluctuations ${ }^{12}$ ), regional connectivity, ${ }^{13,14}$ and global connectivity, ${ }^{15}$ because the VMHC index emphasizes the idea that specific patterns of interhemispheric disconnection can reflect functional consequences for interhemispheric communication or coordination associated with pathological (disease) and physiological conditions. ${ }^{16-18}$ In several studies, abnormal homotopic connectivity in subjects with pathological states has been reported in patients with traumatic axonal injury, ${ }^{17}$ schizophrenia, ${ }^{19}$ autism, ${ }^{20}$ and cocaine addiction. ${ }^{21}$

To our knowledge, despite the evidence of intrinsic functional impairment in PHN, there are no previous studies that have examined voxel-level interhemispheric iFC. In the current study, we hypothesized that the properties of homotopic connectivity would be altered in patients with PHN and that such alteration could be detected using VMHC analyses. Furthermore, this altered VMHC may affect relevant connectivity networks in PHN patients.

\section{Materials and methods}

\section{Participants}

Eighteen right-handed patients with PHN were recruited from the First Affiliated Hospital of Nanchang University. Two consultant physicians from the Pain Department made the clinical diagnoses based on the presence of pain persisting for $>3$ months (93-168 days) after healed herpes zoster infection, defined according to the International Association for the Study of Pain criteria for postherpetic neuralgia. The mean disease duration from the time of disease onset (significant pain according to subjective experience) to the date of the MRI examination was $116.78 \pm 25.96$ days. Patients who had other pain disorders were excluded. The age of the patients with PHN ranged from 46 to 72 years. All the patients reported an obvious case or medical records of shingles associated with varicella zoster virus (VZV) infection, mainly distributed on the unilateral chest and back. The pain of PHN was localized to the left side of the body in 10 cases and to the right side in 8 cases. All the patients accepted mechanical visual analog scale (VAS) evaluation and provided informed written consent prior to undergoing neuroimaging. The pain intensity of all the participants was assessed as at least 7/10 in the VAS records, indicating moderate-to-severe pain (in measurements of pain intensity ranging from 0 to 10 , with 0 indicating no pain and 10 indicating highest tolerable pain). Pain levels were also assessed immediately before functional MRI (fMRI) scanning. These patients had no history of any other major psychiatric illnesses, neurological illnesses, head injuries, or alcohol or drug abuse.

Eighteen right-handed age- and sex-matched healthy controls (HCs) were recruited using advertisements posted in the local community. The age of the HCs ranged from 45 to 70 years. None of the HCs had any significant prior medical diagnoses, substance abuse, neurological disorders, or major psychiatric disorders. Those who had a first-degree relative with a history of severe mental disorder or suicidal behavior were excluded.

Written informed consent was obtained from each participant and the participant's guardian prior to data acquisition. The current study was conducted according to approved guidelines and in compliance with the principles of the Declaration of Helsinki. This study was approved by the Medical Research Ethics Committee and the Institutional Review Board of The First Affiliated Hospital of Nanchang University.

\section{MRI acquisition}

MRI scanning was performed using a 3.0T Siemens TrioTIM Scanner (Erlangen, Bavaria, Germany) at the First Affiliated Hospital of Nanchang University. Each participant underwent both an 8-min resting-state fMRI scan with an echo planar imaging sequence (repetition time $[\mathrm{TR}]=2,000 \mathrm{~ms}$, echo time $[\mathrm{TE}]=30 \mathrm{~ms}$, flip angle $=90^{\circ}$, matrix $=64 \times 64$, field of view $[\mathrm{FOV}]=220 \times 220 \mathrm{~mm}, 4 \mathrm{~mm}$ slice thickness, 30 interleaved axial slices, 240 time-point acquisitions) and a 
three-dimensional, high-resolution T1-weighted structural MRI scan with a magnetization-prepared rapidly acquired gradient echo sequence $(\mathrm{TR}=1,900 \mathrm{~ms}, \mathrm{TE}=2.26 \mathrm{~ms}$, flip angle $=9^{\circ}$, matrix $=240 \times 256$, FOV $=215 \times 230 \mathrm{~mm}$, slice thickness $=1.0 \mathrm{~mm}, 176$ sagittal slices). For resting-state scans, participants were instructed to close their eyes and remain awake while lying quietly and not to think about anything systematically. After the scan, the subjects were asked whether they remained awake during the entire scan.

\section{Resting-state data preprocessing}

Resting-state fMRI images underwent slice timing correction, and voxel-specific head motion calculations and corrections were used to adjust the time series of images using a toolbox for Data Processing \& Analysis of Brain Imaging $^{22}$ (DPABI 2.1; Chinese Academy of Sciences, Beijing, China) that runs on MATLAB 7.14.0 (Mathworks, Natick, MA, USA). Preprocessing comprised the following steps: 1) removal of the first 10 time points; 2) slice timing correction; 3) three-dimensional motion correction; 4) nonlinear registration of the high-resolution $\mathrm{T} 1$ structural images to the Montreal Neurological Institute (MNI) template, in which $\mathrm{T} 1$ structural images were segmented as white matter, gray matter, and cerebrospinal fluid using a new segment algorithm with DARTEL (diffeomorphic anatomical registration through exponentiated lie algebra), ${ }^{23}$ followed by further structural analyses of the resulting segments; 5) spatial normalization to the Montreal Neurological Institute template; 6) resampling of images into a spatial resolution of $\left.3 \times 3 \times 3 \mathrm{~mm}^{3} ; 7\right)$ temporal band-pass filtering (0.01-0.08 Hz); 8) linear detrending and nuisance signal removal (white matter, cerebrospinal fluid, global signal, 6-head motion parameters, 6-head motion parameters at one time point earlier, and the 12 corresponding squared items (Friston 24-parameter model) as covariates) via multiple regression. In this study, we excluded subjects from further analysis if the translation or rotation of head movement was $>2 \mathrm{~mm}$ or $2^{\circ}$ in any direction; the group differences in head motion were evaluated among the patients with $\mathrm{PHN}(\mathrm{n}=18)$ and the HC subjects $(n=18)$ according to the frame-wise displacement (FD) criteria described by Van Dijk et al. ${ }^{24}$

\section{Voxel-mirrored homotopic connectivity} VMHC was computed with a DPABI toolbox. ${ }^{22}$ For each subject, the homotopic connectivity was computed as the Pearson correlation between each pair of mirrored voxel time series. ${ }^{10}$ Fisher's $Z$ transformation was used to improve the normality of the homotopic correlation coefficients. Because $x=0$ defines the brain midline, and there are no voxels medial to this plane, we excluded voxels medial of $x=0$. The resulting correlations were used in the VMHC group-level analyses.

Global and regional group differences in VMHC were examined between the PHN and HC groups. Global VMHC was calculated by averaging the VMHC values across all the voxels within a unilateral hemispheric gray matter mask (there is only one correlation for each pair of homotopic voxels), which was created using the MNI gray matter tissue prior in FSL Version 5.0 (Analysis Group, FMRIB, Oxford UK) (threshold $=25 \%$ tissue-type probability). Group comparisons of global VMHC were performed using twosample $t$-tests. The significance threshold was $P<0.05$, and Bonferroni corrections were used for multiple comparisons. For regional group differences in $\mathrm{VMHC}$, age, sex, and FD values were used as covariates, and the significance threshold correction was based on Gaussian random field (GRF) theory with a voxel level of $P<0.01$ and a cluster level of $P<0.05$. Because FD values can affect the iFC analysis of resting-state fMRI, ${ }^{25,26}$ the mean FD value was applied as a covariate in the group comparisons of VMHC. ${ }^{26}$

The VMHC values of the brain regions that showed abnormal interhemispheric connectivity were extracted, averaged, and correlated using a VAS or the disease duration in the patient group.

\section{Seed-based iFC}

We examined the iFC associated with brain areas that exhibited significantly different VMHC between groups. Specifically, we computed whole-brain voxel-wise Pearson's correlations that were associated with mean time series derived separately for the four regions of interest (ROIs) and comprised all voxels within the prefrontal cortex (PFC) or the precuneus and posterior cingulate cortex (PCUN/PCC) that exhibited reduced VMHC in migraine patients. Fisher $Z$-transformed correlation maps were then entered into a group-level voxel-wise $t$-test analysis, which controlled for age, gender, and FD effects. Subsequently, individual $Z$-values were entered into a random-effect one-sample $t$-test in SPM8 to identify brain regions that showed significantly positive correlations with each seed region for each group with a two-tailed false discovery rate corrected to $P<0.001 .^{27}$ Importantly, in this study, we only considered the brain areas that had positive correlations with each seed region in both the PHN and $\mathrm{HC}$ groups as a mask for the group comparison analysis, because it remains controversial as to whether the anti-correlation is an artifact of the global signal regression in the $\mathrm{iFC}$ analysis. ${ }^{28,29}$ Finally, whole-brain correction for 
multiple comparisons was performed using a preceding united mask (GRF correction, voxel level $P<0.01$ and cluster level $P<0.05)$.

\section{Results}

\section{Demographic and clinical characteristics}

Demographic and clinical information of the participants is listed in Table 1. Two patients with PHN did not complete the MRI scanning, and three patients' data did not pass the imaging data quality control procedure, so these patients were excluded from the final analyses and results. The final sample included 18 patients with $\mathrm{PHN}$ and $18 \mathrm{HCs}$.

\section{Functional homotopy in PHN and HCs}

The spatial patterns of VMHC across the entire gray matter are shown in Figure 1 for the HCs (Figure 1A) and the patients with PHN (Figure 1B). Both groups exhibited the

Table I Participant information

\begin{tabular}{llll}
\hline $\begin{array}{l}\text { Clinical } \\
\text { information }\end{array}$ & PHN (N=18) & HCs (N=18) & $\begin{array}{l}\text { Voxel-wise } \\
\text { t-test } \\
\text { (P-values) }\end{array}$ \\
\hline Age, years & $59.67 \pm 8.4 I$ & $59.27 \pm 7.74$ & $0.144(0.886)$ \\
Sex (male/female) & $11 / 7$ & $11 / 7$ & $0.000(>0.99)$ \\
gVMHC & $0.425 \pm 0.078$ & $0.459 \pm 0.086$ & $-1.215(0.233)$ \\
FD vox, $_{\text {mm }}$ & $0.056 \pm 0.043$ & $0.060 \pm 0.035$ & $-0.281(0.780)$ \\
$\begin{array}{l}\text { VAS score } \\
\text { Disease duration, } \\
\text { days }\end{array}$ & $6.611 \pm 1.470$ & $0.167 \pm 0.383$ & $17.98(0.000 * *)$ \\
\hline
\end{tabular}

Notes: $* * P<0.01$. Data presented as mean $\pm S D$ or number.

Abbreviations: $F D_{\text {vox }}$, voxel-specific frame-wise displacement for in-scanner head motion HCs, healthy controls; NA, not applicable; $\mathrm{PHN}$, postherpetic neuralgia; VAS, visual analog scale; gVMHC, global voxel-mirrored homotopic connectivity. greatest VMHC in the PCUN/PCC, followed by the insula, precentral gyrus, postcentral gyrus, superior temporal cortex, and middle occipital gyrus, along with the subcortical areas. The lateral PFC showed relatively weaker VMHC than other regions in both groups. On visual inspection, although the two groups displayed similar patterns in VMHC, the PHN group seemed to exhibit a weaker VMHC in the PFC and postcentral regions.

\section{Group differences in functional homotopy}

To assess the structural confounders for VMHC, we investigated the global gray matter density differences between the patients with PHN and the HCs. No significant differences were detectable in this measure between the two groups.

The global VMHCs were not significantly different $(t[34]=-1.215 ; P=0.233)$ between the $\operatorname{PHN}(0.425 \pm 0.078)$ and control $(0.459 \pm 0.086)$ groups. However, the local comparisons revealed differences in the dorsolateral prefrontal cortex (DLPFC) and PCUN/PCC, with the PHN group showing reduced $\mathrm{VMHC}$ compared to the control group after controlling for age and sex (GRF correction, voxel-level $P<0.01$ and cluster-level $P<0.05$; Figure 2 and Table 2). None of the brain regions showed stronger $\mathrm{VMHC}$ in the PHN group compared to the control group. The correlation analysis indicated that the VMHC value of the left DLPFC was positively correlated with the GM density of the left DLPFC in the PHN group ( $\rho=0.527 ; P=0.036)$; however, in the right DLPFC ( $\rho=0.286$; $P=0.284)$, left PCUN $(\rho=0.241 ; P=0.368)$, and right PCUN $(\rho=0.265 ; P=0.321)$, there were no such correlations.

A

B

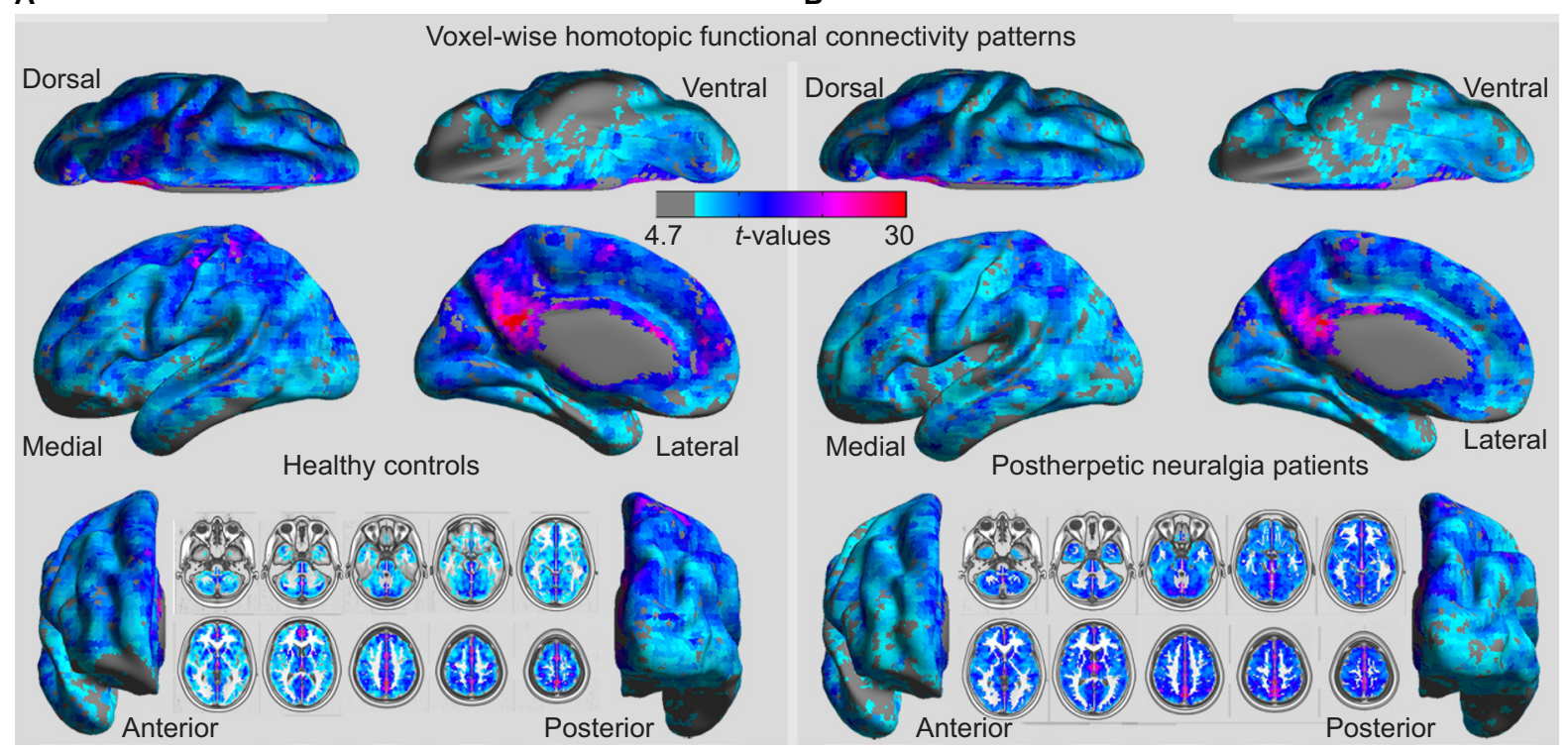

Figure I Whole-brain voxel-wise homotopic functional connectivity patterns in $\mathrm{HCs}(\mathbf{A})$ and patients with $\mathrm{PHN}(\mathbf{B})$.

Abbreviations: $\mathrm{HCs}$, healthy controls; PHN, postherpetic neuralgia. 


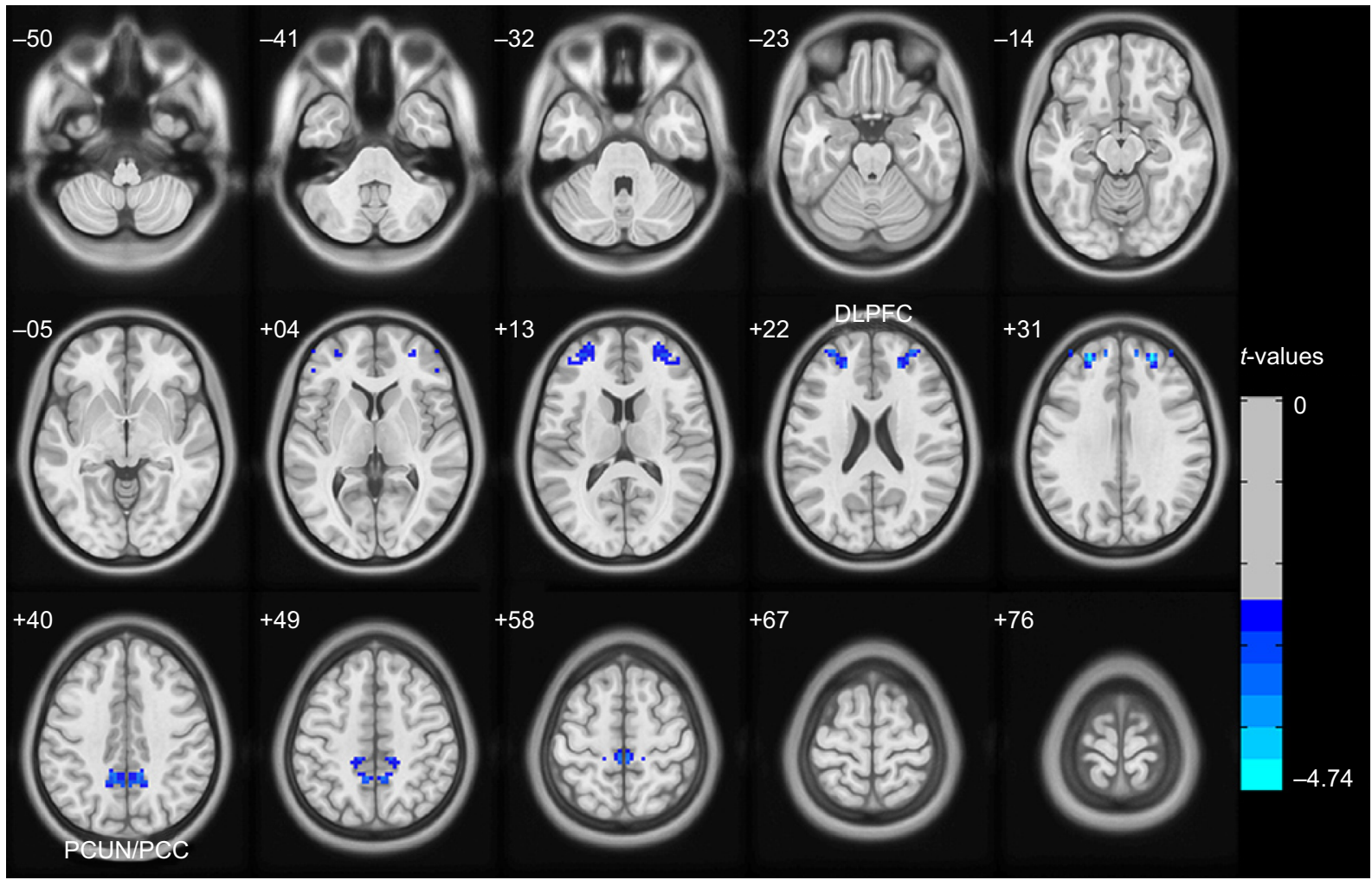

Figure 2 Altered voxel-mirrored homotopic connectivity (VMHC) in patients with postherpetic neuralgia (PHN) compared to healthy controls (HCs).

Notes: The blue colors indicate regions with lower VMHC, which were decreased in patients with PHN in comparison to HCs, as shown after GRF theory correction, with a voxel level of $P<0.01$ and a cluster level of $P<0.05$.

Abbreviations: DLPFC, dorsolateral prefrontal cortex; GRF, Gaussian random field; PCUN/PCC, precuneus and posterior cingulate cortex.

Table 2 Group differences in VMHC between patients with $\mathrm{PHN}$ and $\mathrm{HCs}$ (the values are based on GRF theory correction, with a voxel-level of $P<0.0$ I and a cluster-levelof $P<0.05)$

\begin{tabular}{lllll}
\hline Brain area & BA & $\begin{array}{l}\text { MNI } \\
\boldsymbol{X}, \boldsymbol{Y}, \boldsymbol{Z}\end{array}$ & Peak & Voxel size \\
\hline PHN $<$ HC & & & & \\
DLPFC & 10 & $\pm 24,+48,+30$ & $-4.74 \mathrm{I}$ & $17 \mathrm{I}$ \\
PCUN/PCC & 31.7 & $\pm 6,-5 \mathrm{I},+42$ & -4.477 & 116 \\
\hline
\end{tabular}

Abbreviations: BA, Brodmann area; DLPFC, dorsolateral prefrontal cortex; GRF, Gaussian random field; HCs, healthy controls; MNI, Montreal Neurological Institute; PCUN/PCC, precuneus and posterior cingulate cortex; PHN, postherpetic neuralgia; VMHC, voxel-mirrored homotopic connectivity.

\section{Seed-based iFC}

To evaluate the effects of disrupted interhemispheric connectivity on the relevant resting-state networks, we used clusters that involved the left and right DLPFC and the left and right PCUN/PCC as the four ROI (including all the voxels in these clusters) and investigated patterns of iFC throughout the entire brain in both the PHN and HC groups, as well as group differences in iFC.

The connectivity patterns in $\mathrm{HC}$ participants in the left and right DLPFC and the left and right PCUN/PCC are shown in Figures 3 and S1. The iFC patterns that were seeded in the left and right PFC in the PHN group were very similar, but weaker in strength, to those observed in the $\mathrm{HC}$ group (Figure 3B and E). The group comparisons revealed that patients with PHN showed decreased iFC between the left PFC and the bilateral middle frontal gyrus (MFG) (sup) (Figure 3C and Table 3) and decreased iFC between the right $\mathrm{PFC}$ and the left MFG (Figure $3 \mathrm{~F}$ and Table 3) (GRF corrected, with a voxel-level $P<0.01$ and clusterlevel $P<0.05$ ). None of the reported altered iFC remained in the seed of the left and right PCUN/PCC between the PHN and HC groups after GRF correction (voxel-level $P<0.01$ and cluster-level $P<0.05$ ). In contrast, the results after AlphaSim correction are shown in Figure S1. This alternative approach showed that there was a significant alteration of the iFC in the seed with significantly different VMHC between the groups and a slack corrected condition (Monte Carlo simulation, ${ }^{30}$ single voxel $P=0.05$, FWHM $=6 \mathrm{~mm}, 1,000$ simulations, using every united mask of significantly positive correlations with each seed region in the PHN and $\mathrm{HC}$ groups).

A significant decrease in $\mathrm{iFC}$ was detected between several regions (the left and right cerebellum posterior lobe, right superior/inferior temporal gyrus, left and right PFC, left inferior parietal lobule) and the left PFC (Figure S1B, 

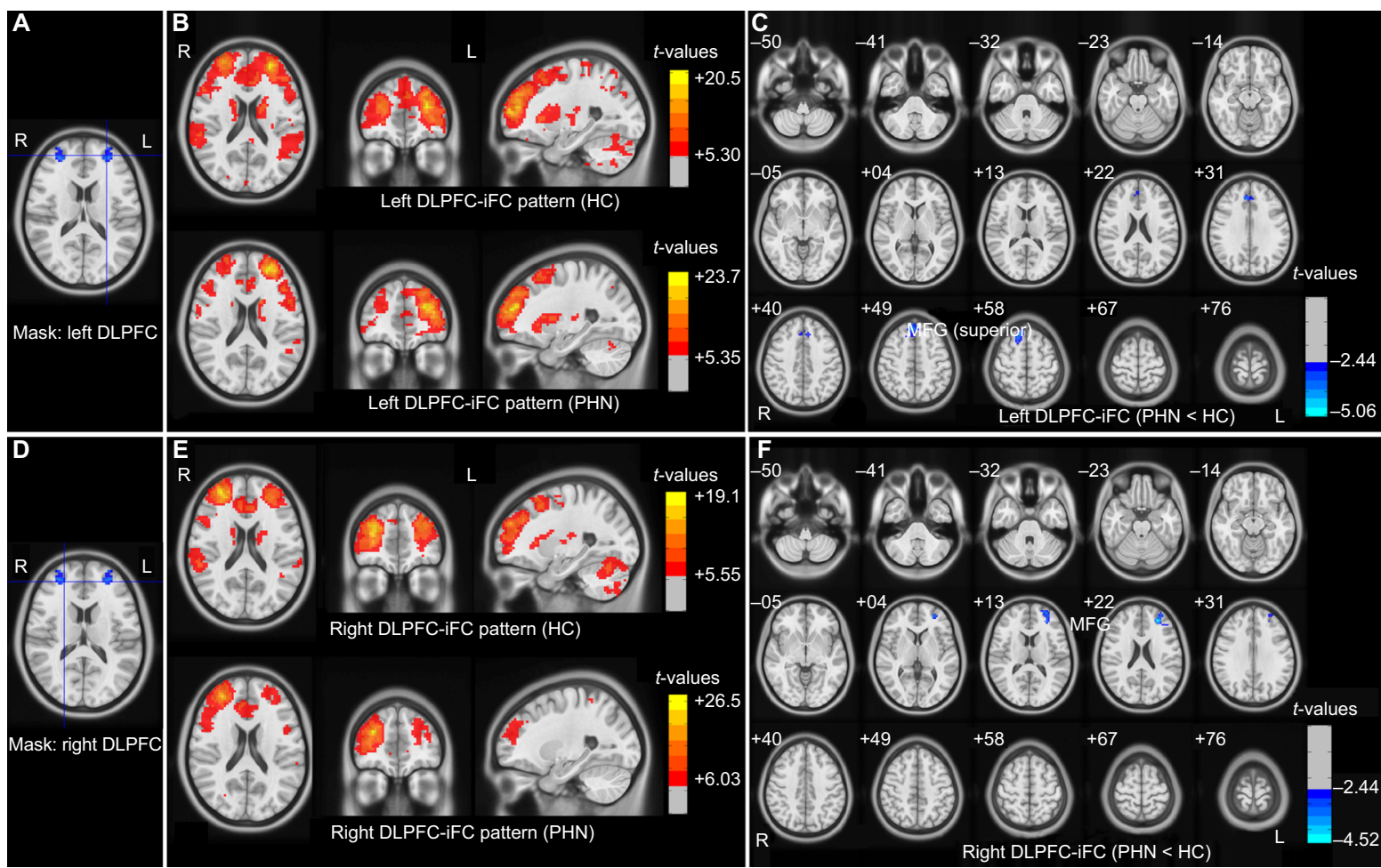

Figure 3 Patterns for iFC for the left and right DLPFC seeds and group differences between the patients with PHN and HCs.

Notes: $(\mathbf{A}$ and $\mathbf{D})$ Seed regions with decreased voxel-mirrored homotopic connectivity in patients with PHN. (B and E) iFC patterns connected with the seed regions in both the PHN and HC groups (with a two-tailed false discovery rate corrected to $P<0.001$ ). ( $G$ and $\mathbf{F}$ ) Group differences in iFC between the two groups (GRF theory correction, with a voxel level of $P<0.0 \mathrm{I}$ and a cluster level of $P<0.05$ ). The color-bars indicate the $t$-values. The figure is presented in radiological convention (the image on the left is the right side of the brain).

Abbreviations: DLPFC, dorsolateral prefrontal cortex; GRF, Gaussian random field; HC, healthy control; iFC, intrinsic functional connectivity; L, left; MFG, middle frontal gyrus; PHN, postherpetic neuralgia; R, right.

Table 3 Group differences in intrinsic functional connectivity between PHN patients and healthy controls (the values are based on GRF theory correction, with a voxel level of $P<0.0$ I and a cluster level of $P<0.05$ )

\begin{tabular}{|c|c|c|c|c|}
\hline Brain area & BA & $\begin{array}{l}\text { Peak (MNI) } \\
X, Y, Z\end{array}$ & Peak & Voxel size \\
\hline \multicolumn{5}{|c|}{ Left DLPFC-FC (PHN < HC) } \\
\hline $\begin{array}{l}\text { Bilateral MFG } \\
\text { (superior) }\end{array}$ & 8,9 & $+3,+39,+45$ & -4.063 & 252 \\
\hline \multicolumn{5}{|c|}{ Right DLPFC-FC $(\mathrm{PHN}<\mathrm{HC})$} \\
\hline Left MFG & 10 & $-27,+42,+21$ & -4.302 & 188 \\
\hline
\end{tabular}

Abbreviations: BA, Brodmann area; DLPFC-FC, dorsolateral prefrontal cortexfrontal cortex; GRF, Gaussian random field; HC, healthy control; MFG, middle frontal gyrus; MNI, Montreal Neurological Institute; PHN, postherpetic neuralgia.

AlphaSim corrected, combined with a different cluster size of $>90$ voxels); between several regions (the left cerebellum posterior lobe, left PFC, left and right inferior parietal lobule, left precuneus) and the right PFC (Figure S1D, AlphaSim corrected, combined with a different cluster size of $>76$ voxels); between the left PFC and the left PCUN/PCC (Figure S1F, AlphaSim corrected, combined with a different cluster size of $>102$ voxels); and between the left PFC and the right PCUN/ PCC (Figure S1H, AlphaSim corrected, combined with a different cluster size of $>99$ voxels).

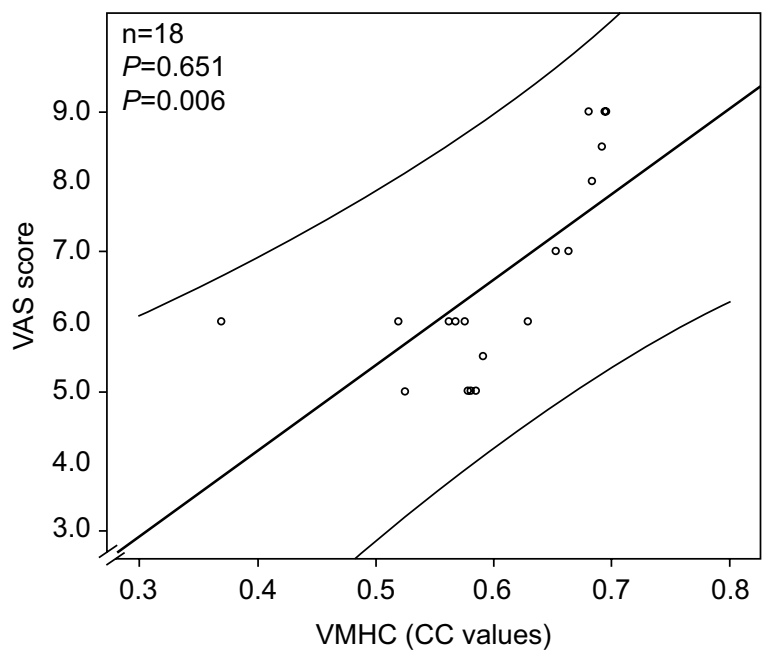

Figure $4 \mathrm{~A}$ significant positive correlation was observed between the $\mathrm{VMHC}$ in the PCUN/PCC and the VAS in the patient group.

Abbreviations: CC, connectivity coefficient; PCUN/PCC, precuneus and posterior cingulate cortex; VAS, visual analog scale; VMHC, voxel-mirrored homotopic connectivity.

\section{Correlations with clinical scores in patients with PHN}

The correlation analysis indicated that the VMHC value of the PCUN/PCC was positively correlated with the VAS of the PHN group ( $\rho=0.651 ; P=0.006$ ) (Figure 4 and Table 4 ). 
Table 4 Correlations between the VMHC/iFC and clinical variables

\begin{tabular}{llllll}
\hline Clinical variables & VMHC & & iFC & \\
\cline { 2 - 3 } & DLPFC $(\rho / P)$ & PCUN/PCC $(\rho / P)$ & & $\begin{array}{l}\text { Between left PFC and } \\
\text { bilateral MFG }(\rho / P)\end{array}$ & $\begin{array}{l}\text { Between right PFC } \\
\text { and left MFG }(\rho / P)\end{array}$ \\
\hline VAS & $0.362 / 0.168$ & $0.651 / 0.006$ & & $0.244 / 0.362$ & $0.201 / 0.460$ \\
Disease duration & $-0.161 / 0.552$ & $-0.211 / 0.433$ & & $-0.150 / 0.578$ & $-0.137 / 0.614$ \\
\hline
\end{tabular}

Abbreviations: DLPFC, dorsolateral prefrontal cortex; iFC, intrinsic functional connectivity; MFG, middle frontal gyrus; PFC, prefrontal cortex; PCUN/PCC, precuneus and posterior cingulate cortex; VMHC, voxel-mirrored homotopic connectivity; VAS, visual analogue scale.

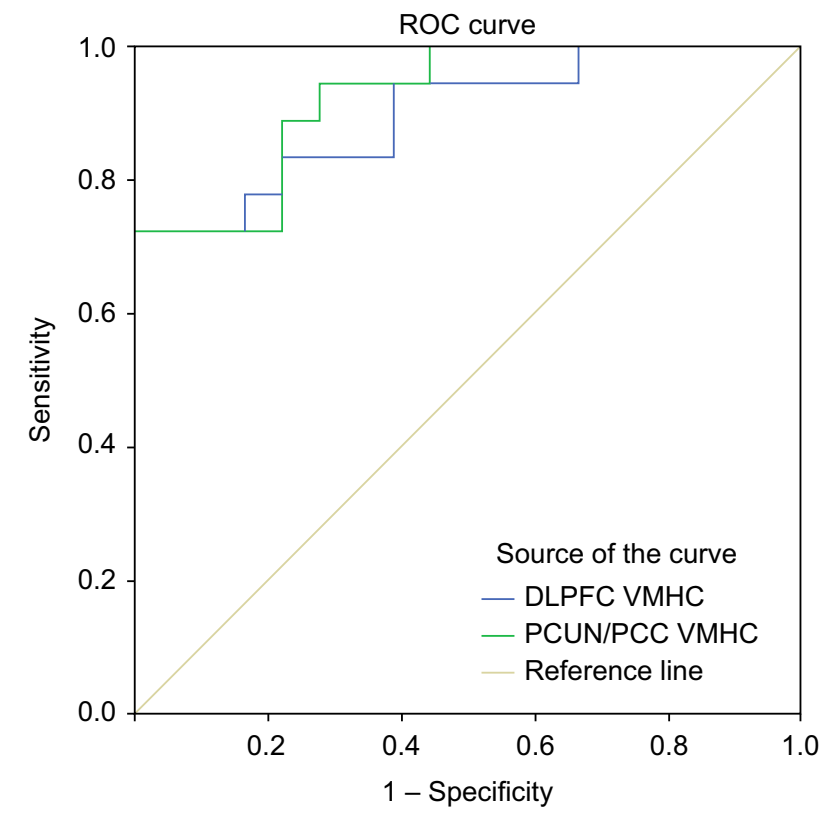

Figure 5 ROC analysis of $\mathrm{VMHC}$ in the altered regions as a potential means to differentiate between patients with PHN and healthy subjects.

Abbreviation: DLPFC, dorsolateral prefrontal cortex; PCUN/PCC, precuneus and posterior cingulate cortex; $\mathrm{PHN}$, postherpetic neuralgia; ROC, receiver operating characteristic; VMHC, voxel-mirrored homotopic connectivity.

\section{Receiver operating characteristic (ROC) analysis of $\mathrm{VMHC}$ in the altered regions between the groups}

As shown above, the MPFC and PCUN/PCC showed significantly different $\mathrm{VMHCs}$ between the groups, which suggested that the VMHC in these two brain regions might be used to discriminate between patients with PHN and healthy subjects. To investigate this possibility, the mean $\mathrm{VMHC}$ values in the two regions were extracted, and then ROC analysis was used. The results revealed that the areas under the curves of the two regions were 0.898 for the PFC and 0.923 for the PCUN/ PCC (Figure 5 and Table S1).

\section{Discussion}

In this study, we obtained the primary finding that PHN-related homotopic connectivity was reduced in the PHN group. The reductions that we identified encompassed the DLPFC and PCUN/PCC, and we also observed decreased PFC-iFC in the MFG. In particular, significant decreases in VMHC in the DLPFC and PCUN/PCC could provide the ability to discriminate between patients with PHN and healthy subjects.

The results from the current study confirm that intrinsic functioning is altered during PHN. First, we observed decreased VMHC in the PCUN/PCC and disrupted PCUN/ PCC related to iFC networks in patients with PHN. The PCUN/PCC, the posterior functional hub of the defaultmode network (DMN), is involved in self-state perception, which combines bottom-up attention with information from memory and perception. ${ }^{31}$ Pain activation in particular is associated with the anterior or middle part of the cingulate cortex $^{32}$ as well as the PCC. ${ }^{33}$ In patients with chronic pain, the PCUN/PCC-related iFC findings indicate that disruptions of the DMN may underlie cognitive and behavioral impairments. ${ }^{34,35}$ In this study, we observed decreased homotopic connectivity in the PCUN/PCC, as well as disrupted iFC networks that are part of the DMN (the anterior components). Furthermore, there was a positive correlation between the VAS and the VMHC value in the PCUN/PCC. These findings suggest that the impairment in the PCUN/PCC in patients with PHN might be relevant for the pain-related impairment that is associated with the cognitive experience of sadness.

In our study, another region with significantly decreased VMHC is the DLPFC, which is the end point for the dorsal pathway (stream) that tells the brain how to interact with stimuli, a process that involves a variety of advanced functions such as executive functions, motor planning, organization, and regulation. In regard to the brain and pain, activation of the DLPFC is associated with cognitive decision making, including pain evaluations and response decisions, and is accomplished by modulation of the corticosubcortical and corticocortical pathways. ${ }^{36}$ Recent noninvasive brain stimulation studies have suggested that interhemispheric DLPFC connectivity influences pain tolerance and discomfort by altering interhemispheric inhibition, ${ }^{37}$ and these studies have also supported the preferential role of the right hemisphere in pain processing because of disrupted homotopic connectivity in the DLPFC. ${ }^{38}$ Microstructural abnormalities in the gray matter of the DLPFC provide a structural foundation for 
understanding the disruption in connectivity, ${ }^{5}$ as iFC is partially restored after pain treatment, which indicates that the DLPFC participates in cognitive task performance and also supports the concept that DLPFC connectivity participates in brain impairment during chronic pain. ${ }^{39}$ In this study, the decreases in VMHC and iFC observed in networks in the DLPFC imply interhemispheric dis-coordination and provide neurodevelopmental evidence for the disconnection hypothesis in PHN.

In patients with PHN, it is clear that the pathological features include peripheral axonal loss and sensory neuronal damage. However, a novel hypothesis is that the pain seen in rats with a latent varicella zoster virus infection could be due to changes in neuronal function that are caused by the mere presence of the virus and that this may also be true of PHN pain. ${ }^{40,41}$ There are reasons to believe that PHN-related deficits in VMHC that result in interhemispheric coordination are caused by viral infection, but this hypothesis needs to be confirmed in the future. VHMC differences between the $\mathrm{PHN}$ and $\mathrm{HC}$ groups might be useful diagnostic markers, and VMHC values in the DLPFC and the PCUN/PCC could help to accurately discriminate between these groups, using a sensitivity of $72.2 \%$ and a specificity of $100 \%$ as cut-off points.

While our results are interesting and encouraging, some limitations of the current study should be noted. First, although we detected interhemispheric functional connectivity differences in patients with PHN and healthy subjects, this study had a relatively small sample size, so a larger sample should be used to confirm our results. Second, while we detected decreased homotopic connectivity in the DLPFC and PCUN/PCC, which are involved in executive control processes, our current study did not use cognitive tasks to investigate the relationship between the neuroimaging findings and the cognitive abilities of the subjects. This relationship should be considered and examined in future studies.

In the current study, we detected reduced homotopic connectivity in the DLPFC and PCUN/PCC and altered iFC patterns in patients with $\mathrm{PHN}$. Moreover, reduced VMHC was associated with VAS scores in the PCUN/PCC. Our study also suggests an important new avenue for exploration to provide further information to better understand the nature of disconnection in the functional architecture of patients with PHN.

\section{Acknowledgments}

The study was supported by grants from the National Natural Science Foundation of China (grant nos. 81260217 and 81460263), Natural Science Foundation of Jiangxi Province (grant no. 2015ZDAB205021), and the Jiangxi
Province Science and Technology Support Program (grant no. 20151BBG70224).

\section{Disclosure}

The authors report no conflicts of interest in this work.

\section{References}

1. Harpaz R, Ortega-Sanchez IR, Seward JF, Advisory Committee on Immunization Practices (ACIP) Centers for Disease Control and Prevention (CDC). Prevention of herpes zoster: recommendations of the Advisory Committee on Immunization Practices (ACIP). MMWR Recomm Rep. 2008;57(RR-5):1-30.

2. Gan EY, Tian EA, Tey HL. Management of herpes zoster and postherpetic neuralgia. Am J Clin Dermatol. 2013;14(2):77-85.

3. Bennett GJ, Watson CP. Herpes zoster and postherpetic neuralgia: past, present and future. Pain Res Manag. 2009;14(4):275-282.

4. Oaklander AL. The density of remaining nerve endings in human skin with and without postherpetic neuralgia after shingles. Pain. 2001;92(1):139-145.

5. Yu B. Microstructural abnormalities in gray matter of patients with postherpetic neuralgia: a diffusional kurtosis imaging study. Pain Physician. 2016;19(4):E601-E611.

6. Geha PY, Baliki MN, Chialvo DR, Harden RN, Paice JA, Apkarian AV. Brain activity for spontaneous pain of postherpetic neuralgia and its modulation by lidocaine patch therapy. Pain. 2007;128(1):88-100.

7. Geha PY, Baliki MN, Wang X, Harden RN, Paice JA, Apkarian AV. Brain dynamics for perception of tactile allodynia (touch-induced pain) in postherpetic neuralgia. Pain. 2008;138(3):641-656.

8. Zhang Y, Liu J, Li L, et al. A study on small-world brain functional networks altered by postherpetic neuralgia. Magn Reson Imaging. 2014;32(4):359-365.

9. Liu J, Hao Y, Du M, et al. Quantitative cerebral blood flow mapping and functional connectivity of postherpetic neuralgia pain: a perfusion fMRI study. Pain. 2013;154(1):110-118.

10. Zuo XN, Kelly C, Di Martino A, et al. Growing together and growing apart: regional and sex differences in the lifespan developmental trajectories of functional homotopy. J Neurosci. 2010;30(45):15034-15043.

11. Stark DE, Margulies DS, Shehzad ZE, et al. Regional variation in interhemispheric coordination of intrinsic hemodynamic fluctuations. J Neurosci. 2008;28(51):13754-13764.

12. Zhou F, Gong H, Liu X, Wu L, Luk KD-K, Hu Y. Increased lowfrequency oscillation amplitude of sensorimotor cortex associated with the severity of structural impairment in cervical myelopathy. Plos one. 2014;9(8):e104442.

13. Zhou F, Xu R, Dowd E, Zang Y, Gong H, Wang Z. Alterations in regional functional coherence within the sensory-motor network in amyotrophic lateral sclerosis. Neurosci Lett. 2014;558:192-196.

14. Zuo XN, Xu T, Jiang L, et al. Toward reliable characterization of functional homogeneity in the human brain: preprocessing, scan duration, imaging resolution and computational space. Neuroimage. 2013;65:374-386.

15. Tomasi $\mathrm{D}$, Volkow ND. Functional connectivity density mapping. Proc Natl Acad Sci U S A. 2010;107(21):9885-9890.

16. Hoptman MJ, Zuo XN, D'Angelo D, Mauro CJ, Butler PD, Milham MP, Javitt DC. Decreased interhemispheric coordination in schizophrenia: a resting state fMRI study. Schizophr Res. 2012;141(1):1-7.

17. Li J, Gao L, Xie K, et al. Detection of functional homotopy in traumatic axonal injury. Eur Radiol. Epub 2016 Apr 5.

18. Bi Y, Yuan K, Feng D, et al. Disrupted inter-hemispheric functional and structural coupling in Internet addiction adolescents. Psychiatry Res. 2015;234(2):157-163.

19. Li HJ, Xu Y, Zhang KR, Hoptman MJ, Zuo XN. Homotopic connectivity in drug-naive, first-episode, early-onset schizophrenia. J Child Psychol Psychiatry. 2015;56(4):432-443. 
20. Anderson JS, Druzgal TJ, Froehlich A, et al. Decreased interhemispheric functional connectivity in autism. Cereb Cortex. 2011;21(5): 1134-1146.

21. Kelly C, Zuo XN, Gotimer K, et al. Reduced interhemispheric resting state functional connectivity in cocaine addiction. Biol Psychiatry. 2011;69(7):684-692.

22. Yan CG, Wang XD, Zuo XN, Zang YF. DPABI: data processing \& analysis for (resting-state) brain imaging. Neuroinformatics. 2016; 14(3):339-351.

23. Goto M, Abe O, Aoki S, et al. Diffeomorphic Anatomical Registration Through Exponentiated Lie Algebra provides reduced effect of scanner for cortex volumetry with atlas-based method in healthy subjects. Neuroradiology. 2013;55(7):869-875.

24. Van Dijk KR, Sabuncu MR, Buckner RL. The influence of head motion on intrinsic functional connectivity MRI. Neuroimage. 2012;59(1):431-438.

25. Power JD, Barnes KA, Snyder AZ, Schlaggar BL, Petersen SE. Spurious but systematic correlations in functional connectivity MRI networks arise from subject motion. Neuroimage. 2012;59(3):2142-2154.

26. Yan CG, Cheung B, Kelly C, et al. A comprehensive assessment of regional variation in the impact of head micromovements on functional connectomics. Neuroimage. 2013;76:183-201.

27. Chumbley JR, Friston KJ. False discovery rate revisited: FDR and topological inference using Gaussian random fields. Neuroimage. 2009;44(1):62-70.

28. Murphy K, Birn RM, Handwerker DA, Jones TB, Bandettini PA. The impact of global signal regression on resting state correlations: are anti-correlated networks introduced? Neuroimage. 2009;44(3): 893-905.

29. Chai XJ, Castanon AN, Ongur D, Whitfield-Gabrieli S. Anticorrelations in resting state networks without global signal regression. Neuroimage. 2012;59(2):1420-1428.

30. Song XW, Dong ZY, Long XY, et al. REST: a toolkit for resting-state functional magnetic resonance imaging data processing. Plos One. 2011;6(9):e25031.
31. Andrews-Hanna JR, Reidler JS, Sepulcre J, Poulin R, Buckner RL. Functional-anatomic fractionation of the brain's default network. Neuron. 2010;65(4):550-562.

32. Shackman AJ, Salomons TV, Slagter HA, Fox AS, Winter JJ, Davidson RJ. The integration of negative affect, pain and cognitive control in the cingulate cortex. Nat Rev Neurosci. 2011;12(3):154-167.

33. Nielsen FA, Balslev D, Hansen LK. Mining the posterior cingulate: segregation between memory and pain components. Neuroimage. 2005;27(3):520-532.

34. Baliki MN, Geha PY, Apkarian AV, Chialvo DR. Beyond feeling: chronic pain hurts the brain, disrupting the default-mode network dynamics. J Neurosci. 2008;28(6):1398-1403.

35. Apkarian AV, Sosa Y, Krauss BR, et al. Chronic pain patients are impaired on an emotional decision-making task. Pain. 2004;108(1-2):129-136.

36. Lorenz J, Minoshima S, Casey K. Keeping pain out of mind: the role of the dorsolateral prefrontal cortex in pain modulation. Brain. 2003;126(5):1079-1091.

37. Rêgo GG, Lapenta OM, Marques LM, et al. Hemispheric dorsolateral prefrontal cortex lateralization in the regulation of empathy for pain. Neurosci Lett. 2015;594:12-16.

38. Sevel LS, Letzen JE, Staud R, Robinson ME. Interhemispheric dorsolateral prefrontal cortex connectivity is associated with individual differences in pain sensitivity in healthy controls. Brain Connect. 2016;6(5):357-364.

39. Čeko M, Shir Y, Ouellet JA, Ware MA, Stone LS, Seminowicz DA. Partial recovery of abnormal insula and dorsolateral prefrontal connectivity to cognitive networks in chronic low back pain after treatment. Hum Brain Mapp. 2015;36(6):2075-2092.

40. Garry EM, Delaney A, Anderson HA, et al. Varicella zoster virus induces neuropathic changes in rat dorsal root ganglia and behavioral reflex sensitisation that is attenuated by gabapentin or sodium channel blocking drugs. Pain. 2005;118(1):97-111.

41. Guedon JM, Yee MB, Zhang M, Harvey SA, Goins WF, Kinchington PR. Neuronal changes induced by Varicella Zoster Virus in a rat model of postherpetic neuralgia. Virology. 2015;482:167-180. 


\section{Supplementary materials}

Table SI ROC analysis for differentiating patients from healthy subjects

\begin{tabular}{lllll}
\hline Reduced connectivity values in patients & Area under the curve & Cut-off point & Sensitivity & Specificity \\
\hline VMHC values in DLPFC & 0.898 & $0.454^{\mathrm{a}}$ & $72.2 \%(13 / 18)$ & $100 \%(18 / 18)$ \\
VMHC values in PCUN/PCC & 0.923 & 0.698 & $72.2 \%(13 / 18)$ & $100 \%(18 / 18)$ \\
iFC values between left PFC and bilateral MFG & 0.883 & 0.356 & $72.2 \%(13 / 18)$ & $94.4 \%(17 / 18)$ \\
iFC values between left PFC and left MFG & 0.824 & 0.409 & $77.8 \%(14 / 18)$ & $72.2 \%(13 / 18)$ \\
\hline
\end{tabular}

Notes: aUsing this cut-off point, the VMHC value of PFC could correctly classify 13 of 18 patients and 18 of 18 healthy subjects, resulting in a sensitivity of $72.2 \%$ and a specificity of $100 \%$. The means for other cut-off points were similar.

Abbreviations: DLPFC, dorsolateral prefrontal cortex; iFC, intrinsic functional connectivity; MFG, middle frontal gyrus; PCUN/PCC, precuneus/posterior cingulate cortex; PFC, prefrontal cortex; ROC, receiver operating characteristic; VMHC, voxel-mirrored homotopic connectivity.

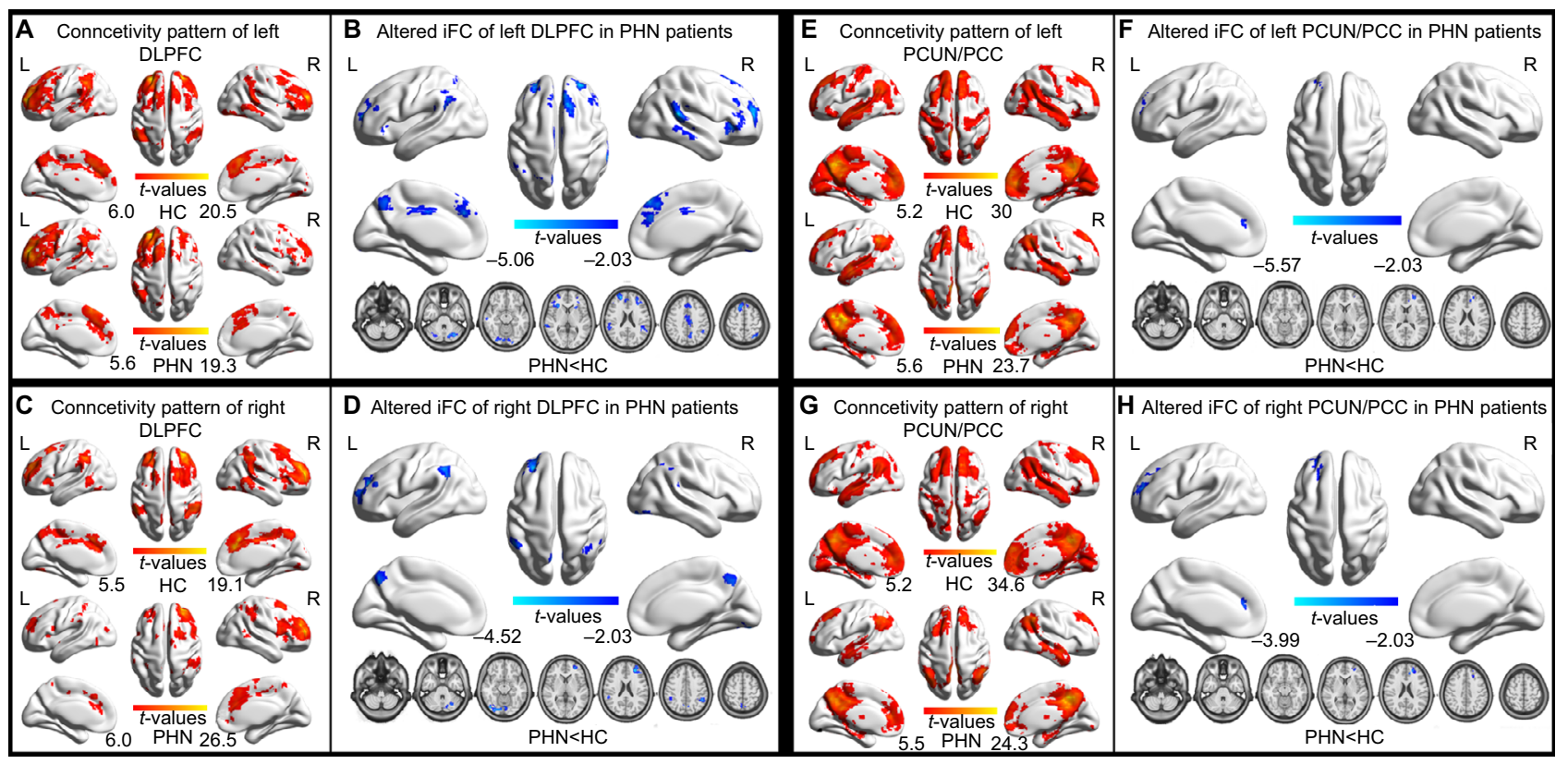

Figure SI Altered iFC patterns between patients with PHN and HCs, with an AlphaSim correction.

Notes: A-H show the iFC patterns of the seed, with significantly different VMHC between the PHN and HC groups (two-tailed FDR corrected to $P<0.00 \mathrm{I}$ ).

Abbreviations: DLPFC, dorsolateral prefrontal cortex; FDR, false discovery rate; HC, healthy control; iFC, intrinsic functional connectivity; L, left; PCUN/PCC, precuneus/ posterior cingulate cortex; PHN, postherpetic neuralgia; R, right.

\section{Publish your work in this journal}

The Journal of Pain Research is an international, peer reviewed, open access, online journal that welcomes laboratory and clinical findings in the fields of pain research and the prevention and management of pain. Original research, reviews, symposium reports, hypothesis formation and commentaries are all considered for publication.
The manuscript management system is completely online and includes a very quick and fair peer-review system, which is all easy to use. Visit http://www.dovepress.com/testimonials.php to read real quotes from published authors. 